\title{
Ueber die Beziehung der Steigzeit und der Steighöhe zur Konzentration beim kapillaren Aufstieg im Filtrierpapier.
}

\author{
Von Hans Schmidt. (Hamburg).
}

(Eingegangen ain 7. Januar 1920.)

In einer früheren Arbeit') konnte ich zeigen, $\mathrm{daB}$ es möglich ist die Konzentration einer verdünnten Säure durch deren Ausbreitung in Lösch- oder Filtrierpapier zu berechnen. In einer späteren Arbeit von mir ${ }^{2}$ ) wurde gezeigt, daß die kapillare Ausbreitung einer verdünnten Mineralsäure in Lösch- und Filtrierpapier den gleichen Gesetzen folgt, ob die Ausbreitung kreisförmig in der Ebene oder als Aufstieg im Streifen erfolgt. In beiden Fällen wird die Berechnung der Konzentration durch den Umstand ermöglicht, daß die Säure durch Ad.' sorption an das Papiermaterial sich weniger ausbreitet als das Wasser, was sich durch einen geeigneten Indikator unschwer feststellen läßt. Diese Möglichkeit der Konzentrationsbestimmung gilt für alle dispersen Systeme, bei denen durch Adsorption die disperse Phase im Aufstieg zurückbleibt; ausgenommen sind natürlich die Fälle elektropositiver Kolloide, die sofort ausflocken und die Filterporen verstopfen (Fichter und $\mathrm{S}$ a h $\mathrm{lb}$ o h m). Während die Konzentrationsbestimmung solcher disperser Systeme an die Wahl geeigneter Indikatoren geknüpft ist, die das Zurückbleiben der dispersen Phase anzeigen, und es demzufolge darauf ankommt, die relative Höhendifferen $z$ bei gleicher Höhe des Dispersionsmittels zu messen ungeachtet der Steigzeit, die (bei geringer Viskosität) nur wenig verschieden zu sein braucht, so läßt dieses Verfahren da im Stich, wo entweder keine besondere Adsorption der dispersen Phase stattfindet, oder dieselbe nicht erkennbar gemacht werden kann. Einige derartige Lösungen können eine mit der Konzentration sehr, wechselnde Steigzeit haben, da letztere ceteris paribus von der Viskosität und diese von der Konzentration abhängt.

Von solchen viskösen Lösungen fand ich für die folgenden Untersuchunger besonders wässerige Lösungen von Glyzerin und Zucker brauchbar, und ich stellte mir die Aufgabe, in dem kapillaren Aufstieg ein Maß für ihre Konzentration zu erhalten.

Die zu den Versuchen benutzten Papiere (Löschpapier 117; Filtrierpapier 602 extra hart, 602 hart, 598 von $C$ Schleicher u. Schüll), sowie die Versuchsanordnung waren die gleichen

\footnotetext{
1) H. Sch midt, Koll.-Zeitschr. 13, 146 (1913).

2) H. Schmidt, Koll-Zeitschr. 24, 19 (1919).
}

wie in meiner früheren Arbeit ${ }^{3}$ ), weswegen auf diese verwiesen sei. Als Material für meine Versuche diente eine Glyzerinlösung, die bei $20^{\circ}$ Celsius ein mittelst Pyknometer ermitteltes spezifisches Gewicht von 1,225 hatte. Diesem Glyzerin entsprach nach W. Gerlach ${ }^{4}$ ) eine Konzentration von 86 Prozent. Während der 10 Tage, in denen die folgenden Versuche gemacht wurden, war anzunehmen, dab das hygroskopische Glyzerin seine Konzentration verändert hatte.

In der Tat ergab eine Nachprüfung ein spezifisches Gewicht von 1,220, dem etwa 84 Prozent entspricht; die daraus hergestellten Verdünnungen hatten sìch entsprechend geändert. Von Zuckerarten wurde Dextrose untersucht, von dem eine bei $20^{\circ}$ Celsius gesättigte, wässerige Lösung hergestellt und als 100 Prozent in Rechnung gesetzt wurde. Daraus wurden dann die weiteren Verdünnungen mit Wasser hergestellt. Die Annahme von 100 Prozent ist willkürlich; das Verhältnis der Konzentrationen der Dextroselösungen untereinander ist jedoch so genau als möglich.

Die folgenden Versuchsprotokolle liegen allen weiteren Rechnungen zugrunde.

Tabelle I.

Glyzerin. Löschpapier 117. Temp. 16-16,50.

\begin{tabular}{|c|c|c|c|c|}
\hline \multirow{2}{*}{$\begin{array}{l}\text { Höhe } \\
\text { in } \mathrm{cm}\end{array}$} & \multicolumn{4}{|c|}{ Zeit in Minuten bei Konzentration in Proz. } \\
\hline & 86 & 57,3 & 28,6 & aqua. \\
\hline 1,25 & 18,7 & 2,6 & 0,66 & 0,25 \\
\hline 2,5 & 63 & 9,5 & 2,36 & 0,88 \\
\hline 3,75 & 122 & 22,2 & 5.36 & 1,91 \\
\hline & 183 & 39,5 & 9,75 & 3,55 \\
\hline 7,5 & 295 & 83,5 & 22,5 & 8,15 \\
\hline 10 & 696 & 120,2 & 40,25 & 14,63 \\
\hline
\end{tabular}

Tabelle II.

Glyzerin. Filtrierpapier 598 (quer geschnitten). Temp. $15-16^{\circ}$.

\begin{tabular}{|c|c|c|c|c|c|c|}
\hline $\begin{array}{l}\text { Höhe } \\
\text { in } \mathrm{cm}\end{array}$ & $\begin{array}{c}\text { Zeit } \\
86\end{array}$ & 57,3 & $\begin{array}{l}\text { ei } K r \\
43\end{array}$ & 28,6 & 17,2 & $\begin{array}{l}\text { Proz. } \\
\text { aqua. }\end{array}$ \\
\hline $\begin{array}{c}2,5 \\
5 \\
7,5 \\
10\end{array}$ & $\begin{array}{r}31,5 \\
104,5 \\
185,5 \\
-\end{array}$ & $\begin{array}{c}4,13 \\
20,5 \\
51,08 \\
93,16\end{array}$ & $\begin{array}{r}2,05 \\
9,16 \\
23,33 \\
44,08\end{array}$ & $\begin{array}{l}1,25 \\
5,73 \\
14,5 \\
28\end{array}$ & $\begin{array}{c}0,46 \\
2,41 \\
6 \\
11,66\end{array}$ & $\begin{array}{l}4,91 \\
9,33\end{array}$ \\
\hline
\end{tabular}

3) H. Schmidt, Koll,-Zeitschr. 24, 49 (1919).

4) W. Gerlach, Chem. Industrie 7, 281 (1884), zitiert in Abderhaldert's Biochem. Handlexikon. 
Tabelle Ill.

Glyzerin. Filtrierpapier 602 hart (quer). Temp. $15,5^{\circ}$

\begin{tabular}{c||c|c|c|c|c|c|}
\hline \hline Höhe & \multicolumn{5}{|c}{ Zeit in Minuten bei Konzentration in Proz. } \\
in $\mathrm{cm}$ & 86 & 57,3 & 43 & 28,6 & 17,2 & aqua. \\
\hline \hline 1,25 & 32 & 4,5 & 1,75 & 1,33 & 0,55 & 0,5 \\
2,5 & 108 & 18,5 & 7,75 & 6 & 2,16 & 2,08 \\
3,75 & 198 & 42,5 & 18,5 & 14 & 1,83 & 4,75 \\
5 & 287 & 73,5 & 33 & 25 & 8,58 & 8,5 \\
7,5 & - & 144,5 & 71 & 55,5 & 19 & 18,83 \\
10 & - & 217 & 118,5 & 97,5 & 34,42 & 33,8
\end{tabular}

Tabelle IV.

Glyzerin. Filtrierpapier 602 extra hart (quer). Temp. $16-17^{\circ}$.

\begin{tabular}{c||c|c|c|c|c|c}
1,25 & 46 & 6,6 & 4,25 & 1,95 & 1,08 & 1,0 \\
2,5 & 164 & 32 & 18 & 9,08 & 4,33 & 3,83 \\
3,75 & 258 & 86,5 & 38,5 & 20,75 & 9,16 & 8,16 \\
5 & 430 & 100 & 62,5 & 36 & 15,5 & 14,16 \\
7,5 & - & 206 & 122 & 78,5 & 34 & 30,0 \\
10 & - & 309,5 & 192,5 & 134,5 & 61 & 51,5
\end{tabular}

Tabelle V.

Dextrose. Löschpapier 117. Temp. 15,2 ${ }^{\circ}$.

\begin{tabular}{c||c|c|c|c|c|c}
\hline \multirow{2}{*}{$\begin{array}{c}\text { Höhe } \\
\text { in cm }\end{array}$} & \multicolumn{6}{c|}{ Zeit in Minuten bei Konzentration in Proz. } \\
100 & 66,6 & 50 & 33,3 & 20 & 12,5 \\
\hline 2,5 & 6,16 & 3,03 & 1,93 & 1,53 & 1,21 & 1,05 \\
5 & 26,5 & 13,5 & 8,91 & 6,66 & 5,33 & 4,5 \\
$.7,5$ & 59 & 31,5 & 21,5 & 15,6 & 12,5 & 10,416 \\
10 & 104,5 & 56,5 & 39,5 & 28,5 & 22,5 & 18,75
\end{tabular}

Tabelle VI.

Dextrose. Filtrierpapier Nr. 598 (quer). Temp. $15^{\circ}$.

\begin{tabular}{c||c|c|c|c|c|c}
\hline \multicolumn{1}{l||}{ Höhe } & \multicolumn{6}{c|}{ Zeit in Minuten bei Konzentration in Proz. } \\
in cm & 100 & 66,6 & 50 & 33,3 & 20 & 12,8 \\
\hline \hline 2,5 & 3,33 & 1,91 & 1,26 & 0,98 & 0,76 & 0,63 \\
5 & 14,5 & 8,41 & 5,66 & 4,33 & 3,5 & 2,75 \\
7,5 & 34,5 & 20,16 & 14 & 10,33 & 8,5 & 6,66 \\
10 & 69,5 & 37 & 26,4 & 19,5 & 15,8 & 12,25
\end{tabular}

Tabelle VII.

Dextrose. Filtriei papier 602 hart (quer). Temp. $15,5^{\circ}$.

\begin{tabular}{c||c|c|c|c|c|c|}
\hline \hline \multicolumn{1}{c||}{ Höhe } & \multicolumn{6}{c|}{ Zeit in Minuten bei Konzentration in Proz. } \\
in cm & 100 & 66,6 & 50 & 33,3 & 20 & 12,5 \\
\hline \hline 1,25 & 2,58 & 1,41 & 1,08 & 0,75 & 0,58 & 0,58 \\
2,5 & 12 & 5,91 & 4,25 & 3,25 & 2,41 & 2,33 \\
3,75 & 27,5 & 14,5 & 10 & 7,66 & 5,91 & 5,5 \\
5 & 47 & 26,5 & 19 & 13,66 & 10,5 & 9,8 \\
7,5 & 100 & 60 & 43 & 30 & 23,75 & 22 \\
10 & - & 106 & 77 & 53 & 42 & 38,5 \\
\end{tabular}

Tabelle VIII.

Dextrose. Filtrierpapier Nr.602 extra hart (quer). Temp. $15-16^{\circ}$.

\begin{tabular}{c||c|c|c|c|c|c|}
\hline \multicolumn{1}{c||}{ Höhe } & \multicolumn{6}{c}{ Zeit in Minuten bei Konzentration in Proz. } \\
in cm & 100 & 66,6 & 50 & 33,3 & 20 & 12,8 \\
\hline \hline 1,25 & 8,5 & 4,38 & 2,7 & 2,45 & 1,61 & 1,23 \\
2,5 & 32 & 18,5 & 12 & 9,5 & 6,83 & 5,25 \\
3,75 & 67 & 40 & 28 & 20,5 & 15 & 11,5 \\
5 & 106 & 60 & 48 & 35,5 & 26 & 19,5 \\
7,5 & 196 & 145 & 102 & 76 & 55 & 40,5 \\
10 & - & 235 & 190 & 135,5 & 102 & 70
\end{tabular}

Beim kapillaren Aufstieg verschieden konzentrierter wässeriger Lösungen von Glyzerin und Dextrose unter sonst gleichen Bedingungen treten drei Größen auf: die Konz. C, die Steighöhe $\mathrm{H}$ und die Steigzeit $t$, welche aufs engste miteinander verknüpft sind, und deren Beziehungen. zueinander im folgenden einzeln untersucht wurden.

I. Beziehung zwischen Steighöhe und Steigzeit bei konstanter Konzentration.

Für die Beziehung der Steighöhe zu der Zeit beim kapillaren Aufstieg einer Flüssigkeit hatten $\mathrm{Cameron}$ und $\mathrm{Bell}^{5}$ ) und unabhängig Wo. Ostwald ${ }^{6}$ ) eine Gleichung gefunden von der Form $H=k \cdot t^{n}$, deren Gültigkeit in späteren Arbeiten von $\mathrm{H}$. Schmidt (loc. cit.) und R. Lucas ${ }^{7}$ ) bestätigt wurde. Für jede Konzentration und für jedes der verwandten Papiere wurde nun die betr. Formel $H=k \cdot t^{n}$ mit Hilfe graphischer Konstruktion berechnet und die Anwendung der Formel ergab auch hier eine gute Uebereinstimmung zwischen gefundenen und beobachteten Werten. Die Werte der Konstanten $\mathrm{k}$ und $\mathrm{n}$ für die einzelnen Gleichungen sind in folgenden Tabellen Ia - VIHa zusammengestellt.

Abweichungen $z$ wischen den von mir und von R. Lucas erhaltenen Zahlen, der mit gleichem Papier gearbeitet hatte, möchte ich dem Umstand zuschreiben, daB meine Versuche bei etwas höherer Temperatur stattfanden. Ueber die Abhängigkeit der Steigzeit und Steighöhe von der Temperatur stehen eingehende Untersuchungen noch aus.

5) Camer on und B e 11, Bull. Nr. 30; Bureau of Soils; W. S. Department of Agriculture; Journal of physic. Chem. 10, 658 (1906).

6) Wo Ostwald, Koll.-Zeitschr. 2, Suppl.-Heft II (1908)

7) R. Lucas, Koll.-Zeitschr. 21, 105, 192 (1917); 23, 15 (1918). 
Tabellen la-IVa. Glyzerin.

\begin{tabular}{|c|c|c|c|c|c|}
\hline & Papier & $\begin{array}{l}\text { Konz. } \\
\text { in Proz. }\end{array}$ & $\mathrm{k}$ & $\mathrm{n}$ & $\begin{array}{c}\text { Mittel- } \\
\text { wert }\end{array}$ \\
\hline$\dot{\text { Ia }}$ & 117 & $\begin{array}{c}86 \\
57,3 \\
28,6 \\
\text { aqua. }\end{array}$ & $\begin{array}{l}0,224 \\
0,758 \\
1,660 \\
2,754\end{array}$ & $\begin{array}{l}0,58 \\
0,51 \\
0,479 \\
0,48 \\
\end{array}$ & 0,5 \\
\hline II a & 598 & $\begin{array}{c}86 \\
57,3 \\
43 \\
28,6 \\
17,2 \\
\text { aqua. }\end{array}$ & $\begin{array}{l}0,276 \\
1,318 \\
1,799 \\
2,291 \\
3,467 \\
3,758\end{array}$ & $\begin{array}{l}0,618 \\
0,450 \\
0,454 \\
0,444 \\
0,439 \\
0,439\end{array}$ & 0,44 \\
\hline III a & $602 \mathrm{~h}$. & $\begin{array}{l}86 \\
57,3 \\
43 \\
28,6 \\
17,2 \\
\text { aqua. }\end{array}$ & $\begin{array}{l}0,141 \\
0,586 \\
0,929 \\
1,047 \\
1,667 \\
1,742\end{array}$ & $\begin{array}{l}0,616 \\
0,5 \\
0,49 \\
0,49 \\
0,50 \\
0,50\end{array}$ & 0,5 \\
\hline IVa & 602 e. h. & $\begin{array}{l}86 \\
57,3 \\
43 \\
28,6 \\
17,2 \\
\text { aqua. }\end{array}$ & $\begin{array}{l}0,204 \\
0,478 \\
0,549 \\
0,794 \\
1,12 \\
1,239\end{array}$ & $\begin{array}{l}0,5444 \\
0,500 \\
0,578 \\
0,517 \\
0,538 \\
0,531\end{array}$ & 0,53 \\
\hline
\end{tabular}

Tabellen Va-VIIIa.

Dextrose.

\begin{tabular}{|c|c|c|c|c|c|}
\hline & Papier & $\begin{array}{l}\text { Konz. } \\
\text { in Proz. }\end{array}$ & $\mathrm{k}$ & $\mathrm{n}$ & $\begin{array}{c}\text { Mittel- } \\
\text { wert }\end{array}$ \\
\hline $\mathrm{Va}$ & 117 & $\begin{array}{c}100 \\
66,6 \\
50 \\
33,3 \\
20 \\
12,5\end{array}$ & $\begin{array}{l}0,955 \\
1,429 \\
1,738 \\
2,028 \\
2,16 \\
2,34\end{array}$ & $\begin{array}{l}0,51 \\
0,48 \\
0,47 \\
0,47 \\
0,50 \\
0,50\end{array}$ & 0,5 \\
\hline VIa & 598 & $\begin{array}{l}100 \\
66,6 \\
50 \\
33,3 \\
20 \\
12,5 \\
\end{array}$ & $\begin{array}{l}1,429 \\
1,841 \\
2,239 \\
2,559 \\
2,818 \\
3,141 \\
\end{array}$ & $\begin{array}{l}0,46 \\
0,47 \\
0,46 \\
0,46 \\
0,46 \\
0,47 \\
\end{array}$ & 0,463 \\
\hline VIÍa & 602 h. & $\begin{array}{c}100 \\
66,6 \\
50 \\
33,3 \\
20 \\
12,5\end{array}$ & $\begin{array}{l}0,662 \\
1,023 \\
1,202 \\
1,380 \\
1,517 \\
1,585\end{array}$ & $\begin{array}{l}0,51 \\
0,49 \\
0,49 \\
0,49 \\
0,50 \\
0,50 \\
\end{array}$ & 0,5 \\
\hline VIII a & 602 e. h. & $\begin{array}{c}100 \\
66,6 \\
50 \\
33,3 \\
20 \\
12,5\end{array}$ & $\begin{array}{l}0,371 \\
0,616 \\
0,691 \\
0,794 \\
0,977 \\
1,094\end{array}$ & $\begin{array}{l}0,56 \\
0,50 \\
0,47 \\
0,51 \\
0,50 \\
0,52\end{array}$ & 0,5 \\
\hline
\end{tabular}

Die empirisch gefundene Formel $\mathrm{H}=\mathrm{k}$. $\mathrm{t}^{\mathrm{n}}$ ist dem Vorgang des kapillaren Aufstieges zwar sehr angepaßt, aber sie ist nicht genügend eindeutig in bezug auf die Konstanten $\mathrm{k}$ und $\mathrm{n}$, über die weiter nichts ausgesagt wird.
Einer Arbeit von. R. Lucas (loc. cit.) zufolge gibt es eine Gleichung, aus der die Natur der Konstanten $\mathbf{k}$ hervorgeht.

Setzt man in $\mathrm{H}=\mathrm{k} \cdot \mathrm{t}^{\mathrm{n}}$

Dann wird

$$
\begin{aligned}
K & =\mathrm{k}^{\frac{1}{n}} \\
H^{\frac{1}{n}} & =K \cdot t
\end{aligned}
$$

Bezeichnet man mit

$\omega$ die Oberflächenspannung.

$\eta$ die Zähigkeit,

$r$ den Radius der Kapillaren des Papiers (die kreisförmig im Querschnitt gedacht sind),

d den Randwinkel bei nicht vollkommener Benetzung,

so wird, unter Voraussetzung, daß $\mathrm{H}$ gegenüber der maximalen Steighöhe klein ist,

$$
\mathrm{H}^{2}=\frac{m}{\eta} \cdot \frac{\mathrm{r}}{2} \cdot \cos \mathrm{d} \cdot \mathrm{t} .
$$

Diese Gleichung geht in die obige Formel von Ostwald über, wenn man

$$
\begin{aligned}
\mathrm{n} & =0,5 \text { und } \\
\mathrm{K} & =\frac{\omega}{\eta} \cdot \frac{\mathrm{r}}{2} \cos \mathrm{d} \text { setzt. }
\end{aligned}
$$

In den Tabellen Ia-VIII a bewegen sich die Zahlen sämtlicher $n$-Werte so nahe um 0,5 herum, daß man annehmen kann, daß allen Gleichungen der gleiche Wert 0,5 für $\mathrm{n}$ zukommt. (Es wiirde sich also um Parabeln handeln.)

Die Uebereinstimmung ist bei Dextrose genauer, da auch den Messungen mit Dextrose weniger Fehler zugrunde liegen, wie mit dem durch sein wasseranziehendes Vermögen vetänderlichen Glyzerin. Auch dürfte die nicht genau gleiche Temperatur, bei der die Versuche stattfanden, für die eine oder andere Abweichung verantwortlich zu machen sein.

Sofern es sich bei Glyzerin und Zucker um Stoffe handelt, die die Oberflächenspannung des Wassers gegen Luft nicht wesentlich herabsetzen, so bleibt eine Aenderung der Oberflächenspannung bèi Aenderung der Konzentration unbemerkbar und für Glyzerin und Zucker in wässeriger Lösung ist praktisch 10 als konstant $z u$ betrachten.

Unter dieser Voraussetzung und bei Verwendung wässeriger Lösungen und gleichem Papier ist demnach der Ausdruck

$$
\left(\omega \cdot \frac{r}{2} \cdot \cos d\right)
$$

praktisch von der Konzentration unabhängig und als konstant $z u$ betrachten.

Es wäre demnach 


$$
\begin{aligned}
\mathrm{k}^{\frac{1}{n}}=\mathrm{K} & =\frac{\text { konst. }}{\eta} \\
\text { oder } \eta \cdot \mathrm{K} & =\text { konst. }
\end{aligned}
$$

Dies ist die Gleichung einer rechtwinkligen Hyperbel. In den Gleichungen der Tabellen Ia --Vlll a repräsentieren demnach die Werte für die Konstante $\mathrm{k}$ die Faktoren, die den Unterschied in der Steigzeit für die gleiche Höhe bewirken. Der wesentlichste Faktor ist erfahrungsgemäß die Viskosität, die nach obiger Formel dem Wert fur $\mathrm{k}$ umgekehrt proportional zu setzen ist.

Bei Bestimmung der Viskosität von Glyzerin und Dextrose habe ich mich nicht des üblichen Viskosimeters nach W. Ostwald bedient, da die Ausflußzeiten bei Zimmertemperatur für Glyzerin zu groß wurden, sondern ich benutzte das kleine, nicht gebogene Modell des Stalagmometers von Traube für die Bestimmung der Oberflächenspannung von viskösen Flüssigkeiten und bestimmte die Ausflufzeit derjenigen Flüssigkeitsmenge, die in dem durch Marken abgegrenzten Volumen sich befand. Bezeichnet $\eta$ đie Zähigkeit und $z$ die beobachtete Ausflußzeit, bei konstanter Temperatur, dann gilt für diese Temperatur

$$
\eta: \eta_{\omega}=\mathrm{z}: \mathrm{z}_{\boldsymbol{\omega}} .
$$

Die Temperatur betrug $19^{\circ}$ Celsius und nach Thorpe und $\operatorname{Rodger}^{8}$ ) beträgt für Wasser

$$
\eta_{\omega}=0,0100 \text {. }
$$$$
\text { Demnach } \quad \eta=\frac{\mathrm{z}}{\mathrm{z}_{\omega}} \cdot 0,0100 \text { : }
$$

Die Messungen bei Dextrose waren bei einer Temperatur von 17,8 gemacht. Für diese Temperatur ist $\eta_{\omega}=0,0110$. Die so erhaltenen Werte sind in folgenden Tabellen IX und $X$ gegeben.

Für Flüssigkeiten, die ihre Oberflächenspannung nicht merklich mit der Konzentration ändern, ergab sich (bei konstanter Temperatur)

$$
\mathrm{K} \cdot \eta=\text { konst. }
$$

Legt man den Rechnungen die Beobachtungen bei Papier 602 hart (Tab. III und VII) zugrunde, die der Bedingung $\mathrm{n}=0,5$ am nächsten kommen, so zeigen folgende Tabellen XI und XII wenigstens für Dextrose eine befriedigende Konstanz, während die Abweichungen für Glyzerin beträchtlicher sind.

Es muB jedoch berücksichtigt werden, daf sowohl den Konzentratıonswerten wie auch den (1894).

8) Thorpe and Rodger, Proc. Roy. Soc. 55, 148
Viskositätsbestimmungen keine große Genauigkeit zukommt; auberdem sind noch die bei allen Kapillarsteigversuchen nur schwer vermeidbaren Beobachtungsfehler in Betracht $z u$ ziehen.

Tabelle IX.

Glyzerin bei $19^{\circ}$.

\begin{tabular}{l|c|c}
\hline $\begin{array}{c}\text { Konz. } \\
\text { in Proz. }\end{array}$ & $\begin{array}{c}\text { Ausflußzeit } \\
\text { in Minuten }\end{array}$ & $\eta$ \\
\hline 86 & 70 & $8578,0 \times 10^{-4}$ \\
$57,3 *$ & - & 1300,0 \\
43 & 2,83 & 349,2 \\
32,25 & 1,90 & 232,8 \\
$28,6^{*}$ & - & 209,0 \\
24,8 & 1,53 & 187,4 \\
19,1 & 1,23 & 150,7 \\
$17,2 *$ & - & 142,5 \\
12,45 & 1,05 & 128,6 \\
6,8 & 0,93 & 110,2 \\
4,3 & 0,88 & 107,8 \\
aqua. & 0,816 & 100,0
\end{tabular}

Durch graphische Interpolierung ermittelt.

Tabelle X.

Dextrose bei $17,8^{\circ}$.

\begin{tabular}{c|c|c}
\hline $\begin{array}{c}\text { Relative } \\
\text { Konz. } \\
\text { in Proz. }\end{array}$ & $\begin{array}{c}\text { Ausflußzeit } \\
\text { in Minuten }\end{array}$ & $\eta$ \\
\hline 100 & 17,20 & $1081,0 \times 10-4$ \\
80 & 8,95 & 562,5 \\
66,6 & 5,95 & 374,0 \\
50 & 4,08 & 256,0 \\
33,3 & 2,91 & 182,8 \\
20 & 2,33 & 146,4 \\
aqua. & 1,75 & 110,0
\end{tabular}

Tabelle XI.

Glyzerin. Papier 602 hart.

\begin{tabular}{l|l|l}
\hline $\begin{array}{c}\text { Konz. } \\
\text { in Proz. }\end{array}$ & $\mathrm{k}^{2}=\mathrm{K}$ & $\eta \cdot \mathrm{K}$ \\
\hline & 0,0198 & $170,7 \times 10^{-4}$ \\
86 & 0,343 & 446,0 \\
57,3 & 0,864 & 301,7 \\
43 & 1,096 & 229,0 \\
28,6 & 2,778 & 395,8 \\
17,2 & 3,034 & 303,4 \\
aqua. &
\end{tabular}

Tabelle XII.

Dextrose. Papier 602 hart.

\begin{tabular}{c|c|c}
\hline $\begin{array}{c}\text { Konz. } \\
\text { in Proz. }\end{array}$ & $\mathrm{k}^{2}=\mathrm{K}$ & $\mathrm{K} \cdot \eta$ \\
\hline 100 & 0,438 & $473,4 \times 10^{-4}$ \\
66,6 & 1,046 & 391,2 \\
50 & 1,444 & 370,5 \\
33,3 & 1,904 & 346,5 \\
20 & 2,301 & 336,8 \\
aqua. & 3,034 & 333,7
\end{tabular}


Stellt man die Beziehung zwischen der Konzentration und den Werten für $\mathrm{k}$ aus der Formel $\mathrm{H}=\mathrm{k} \cdot \mathrm{t}^{0,5}$ graphisch dar, so findet man, daß gemäb den Fehlerquellen das Kurvenbild für Dextrose ein genaueres wird als das für Glyzerin. Fig. 1 stellt die $\mathrm{C}-\mathrm{k}$. Kurve für Dextrose dar.

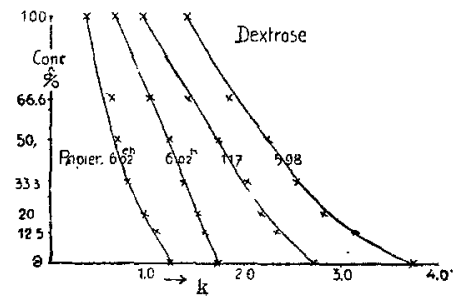

Fig. 1

Die verschiedenen Papiersorten ordnen sich in die Kurvenschar nach dem Grade ihrer Durchlässigkeit ein, resp. nach der Geschwindigkeit, mit der eine bestimmte Höhe vom Wasser erreicht wird. Es ist die gleiche Reihenfolge, die R. Luc as für die Filtriergeschwindigkeit und für die Porengröße fand. - Für eine bestimmte Konzentration sind die Werte von $k$ um so größer, je poröser das Papier ist, und umgekehrt. Mit abnehmender Konzentration wächst bei ein und demselben Papier der Wert für $\mathrm{k}$ und ist am größten bei Wasser. Der Konzentrationsbereich zwischen Wasser einerseits und der gesättigten Dextroselösung $\mathrm{pzw}$. einer 100 prozentigen Glyzerinlösung anderseits ist nur ein Ausschnitt aus der Kurve, die vielmehr nach beiden Seiten weiter verlaufend gedacht werden muß. Die Kurven nähern sich der Konzentrationsachse immer mehr und werden sie schlieblich in einem Punkte treffen, wo $\mathrm{k}=\mathrm{o}$ ist, resp, da $\mathrm{k}$ umgekehrt proportional der Viskosität ist, wo die Viskosität einen solchen Wert erreicht hat, bei dem ein Aufsteigen im Papier überhaupt nicht mehr stattfinden kann. - Die Schnittpunkte mit der k-Achse sind insofern willkürlich, als die $\mathrm{k}$-Achse durch die Konzentration o resp. Wasser gelegt wurde, mithin die Schnittpunkte die $k$-Werte für Wasser darstellen. Die Kurve ist jedoch darüber hinaus verlängert und muß sich einer Geraden nähern, die einer Flüssigkeit von denkbar geringster Viskosität, demnach größtem $\mathrm{k}$-Wert entspricht.

Würde man demnach statt der Konzentration die Viskosität als Ordinate nehmen, dann würde man eine graphische Beziehung zwischen den $\mathrm{k}$-Werten der Papiere und der Viskosität für alle Werte von $\eta$ erhalten. $D a$ die Steig- geschwindigkeitskonstante $\mathrm{k}$ auch vom Radius $\mathrm{r}$ abhängt, so hat man auch eine graphische Darstellung der Steigzeit für Kapillarsysteme aller Grade, vom dichtesten Papier an bis zur weitestèn Glaskapillare. Diese Kurven würden dann den kinetischen Vorgang beim kapillaren Aufstieg im Filtrierpapier für eine bestimmte Flüssigkeit bei gleicher Temperatur vollständig wiedergeben; vorausgesetzt dabei ist, $\mathrm{daB}$ die Oberflächenspannung sich mit Zunahme der Konzentration nicht merklich ändert.

Durch die Oberflächenspannung witd nur die maximale Steighöhe beeinflußt, die jedoch mit Papier experimentell nur angenähert darzustellen ist wegen verschiedener Umstände, darunter auch dem, daß gute Filtrierpapiere im Handel nicht in Streifen zu erhalten sind, die lang genug sind.

Setzt man zu Wasser etwas Saponin- oder Essigsăure, wodurch die Oberflächenspannung des Wassers gegen Luft stark vernindert wird, so findet sich kein Unterschied von reinem Wasser in bezug auf die Zeit, die die Flüssigkeit braucht, um eine bestimmte Höhe zu erreichen.

II. Beziehung von Konzentration zur Höhe bei konstanter Zeit.

Sehr ähnlich den $C-k$-Beziehungen sind diejenigen $z$ wischen der Konzentration der Lösung zu den Höhen, die in gleichen Steigzeiten erreicht werden. Die Höhenwerte wurden durch graphische Interpolierung ermittelt aus den Daten der Versuche I-VIII und in folgendem sei eins dieser Protokolle in Tab. XIII wiedergegeben.

Tabelle XIII.

Dextrose. Papier 117.

\begin{tabular}{|c|c|c|c|c|}
\hline \multirow{3}{*}{$\begin{array}{c}\text { Konz. } \\
\text { in Proz. }\end{array}$} & \multicolumn{4}{|c|}{ Steigzeit in Minuten } \\
\hline & 10 & 20 & 30 & 60 \\
\hline & \multicolumn{4}{|c|}{ Höhe in $\mathrm{cm}$} \\
\hline 100 & 3,125 & 4,25 & 5,25 & 7,65 \\
\hline 66,6 & 4,25 & & 7,2 & 10,25 \\
\hline 50 & 5,25 & 7,25 & 8,75 & 12,5 \\
\hline 33,3 & 5,75 & 8,5 & 10,25 & 14,37 \\
\hline 20 & 6,7 & 9,5 & 11,62 & 一 \\
\hline $12 ; 8$ & 7,3 & 10,35 & 12,65 & - \\
\hline aqua. & 8,375 & 11,4 & 13,62 & 18,12 \\
\hline
\end{tabular}

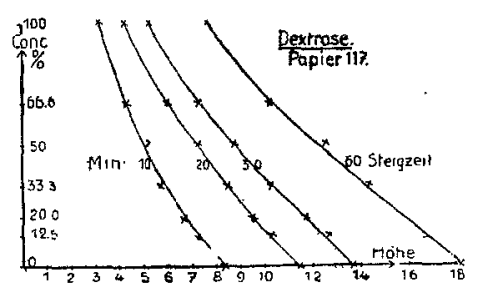

Fig. 2

Die graphische Darstellung in Fig. 2 läßt die große Aehnlichkeit erkennen mit dem 
Kurvenbild der Fig. 1. Auch hier ist die Kurve nach beiden Seiten verlängert zu denken und nähert sich wie dort mit steigender Konzentration einem Maximum an Viskosität, für das $t=0$ wird und nach der anderen Seite einem geometrischen Ort für das Minimum der Viskosität, für das $t$ die oberste Grenze wird. (Gase?)

Genauere Untersuchungen, bei denen statt der Konzentration die Viskosität aufgetragen wird, werden die Beziehungen zwischen der $\mathrm{C}-\mathrm{k}$ - und der $\mathrm{C}-\mathrm{H}$-Kurve besser mathematisch erfassen lassen, und würden dazu beitragen, die Viskosität in klare Beziehung zu bringen zu dem komplexen Phänomen des kapillaren Aufstieges in Papieren.

III. Die Beziehung zwischen Konzentration und Zeit bei konstanter Höhe.

Nimmt man an, daß eine ähnliche Beziehung, wie zwischen $\mathrm{H}$ und $\mathrm{t}$ bei konstanter Konzentration in Form der Gleichung $\mathrm{H}=\mathrm{k} . \mathrm{t}^{\mathrm{n}}$ besteht, auch für die Beziehung von $\mathrm{C} z u$ t bei gleicher Höhe gilt, so würden die Logarithmen der Werte von $C$ und $t$ in eine gerade Linie zu liegen kommen. Die Fig. 3 läbt eine solche Beziehung als möglich erscheinen, aber, wie die Fig. 4 zeigt, kommen die logarithmiertenWerte anstatt in eine gerade Linie in eine ähnliche Kurve zu liegen. Die Beziehung von $\mathrm{C}$ zu $t$ bei konstanter Höhe muß demnach anderer Natur sein.

Bei Analysierung der Kurve ergibt sich jedoch eine Möglichkeit, unter bestimmter Annahme doch eine ähnliche Beziehung zu erhalten, die eine numerisch ziemlich gute Uebereinstimmung zwischen Berechnung und $\mathrm{Be}$ obachtung liefert.

Die Glyzerinkonzentration schwankt zwischen 100 und 0 Prozent. - Das gleiche gilt für Dextrose unter Berücksichtigung der oben erwähnten willkürlichen Festsetzung einer bei Zimmertemperatur gesättigten Dextroselösung gleich 100 Prozent. - Praktisch ist aber schon 1 Prozent in bezug auf Steighöhendifferenz bei gleichen Zeiten nicht mehr zu unterscheiden. Das für solche Messungen in Betracht kommende Konzentrationsbereich wäre dann zwischen 100. Prozent und 1 Prozent, $r 1 \ldots . . . . . .$. Logarithmen derselben 2 und $U$ als urenzen eingeschlossen.

In Fig. 3 verläuft die Kurve für $\mathrm{H}=5$ folgendermaken. Die Kurve beginnt bei der Konzentration 0 in einem Punkte der t-Achse, der dem Wert für die Steigzeit für Wasser bis zur Höhe $H=5$ entspricht. Nach steilem Verlauf im Antang flacht sich die Kurve ab und nähert sich allmählich einem bestimmten Wert für $\mathrm{t}$, der einer Konzentration von 100 Proz. entspricht. Dieser Wert ist ein endlicher, und die Kurve läuft für Glyzerin nicht weiter. Der asymptotische Verlauf ist nur ein scheinbarer. Die Kurve ist vielmehr von zwei bestimmten Punkten begrenzt.

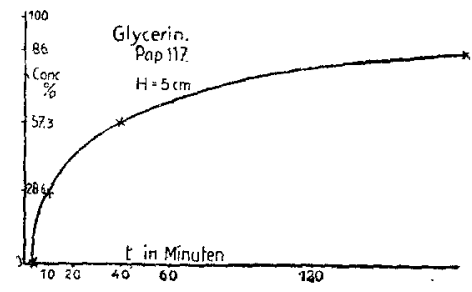

Fig. 3

Die graphische Darstellung der Logarithmen von $C$ und $t$ in Fig. 4 gibt für den gleichen Fall folgenden Verlauf :

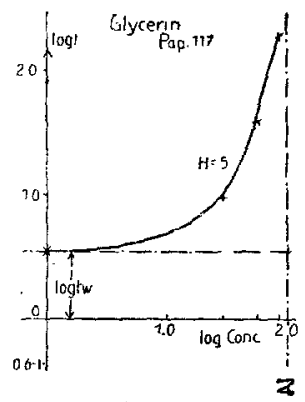

Fig. 4

Die Kurve beginnt bei der höchsten Konzentration $(\log$ Konz. $=2,0)$. Die Kurve verläuft anfangs steil, dann flacher werdend und nähert sich scheinbar asymptotisch einer Geraden, die parallel zur $(\log$ Konz.)-Achse ist und für welche gilt $\log t=$ konstant. $t$ ist hier der Wert für Konzentration 0 Prozent, also für Wasser, und bedeutet die Steigzeit für Wasser bis zur Höhe $\mathrm{H}=5$. Da der Endpunkt der Kurve bei demjenigen $\log \mathrm{t}$-Wert liegt, der der Konzentration 0 Prozent entspricht, letzterer Logarithmus aber nicht darstellbar ist, so ist der Verlauf der Kurve tatsächlich asymptotisch. Praktisch ist aber, wie gesagt, schon 1 Prozent n..... gleich Wasser zu setzen, und der schnittpunkt der Kurve mit der $\log t$-Achse gibt den Wert für $t$, den Wasser für die betreffende Steighöhe hat. Nimmt man daher diesen Schnittpunkt als Koordinatenanfang, transformiert man also die log $t$-Werte zu $\left(\log t-\log t_{\omega}\right)$, dann kann man eine ähnliche 
Beziehung wie oben $\mathrm{H}=\mathrm{k} \cdot \mathrm{t}^{\mathrm{n}}$ hier zwischen $\log C$ und $\left(\log t-\log t_{(0)}\right)$ annehmen. Es bestände also die Gleichung:

$$
\log \mathrm{C}=\beta \cdot\left(\log t-\log \mathrm{t}_{\omega}\right)^{\gamma} .
$$

Mithin müBte die graphische Darstellung der Gleichung $\log (\log C)=\log \beta+\gamma \log \left[\log t-\log t_{\omega}\right]$ eine gerade Linie geben.

Diese Gleichung wurde auf die Versuchsprotokolle I - VIII angewandt und im folgenden sei der Raumersparnis halber nur das Protokoll für den Versuch I gegeben in den Tab. Ib und I $\mathrm{c}$.

Tabelle Ib.

\begin{tabular}{c|c|c|c|c|c|c|c}
\hline $\begin{array}{c}\text { Konz. } \\
\text { in Proz. }\end{array}$ & $\begin{array}{c}\mathrm{H}= \\
\log \text { Konz. }\end{array}$ & $\begin{array}{c}1,25 \\
\log \mathrm{t}\end{array}$ & $\begin{array}{c}2,5 \\
\log \mathrm{t}\end{array}$ & $\begin{array}{c}3,75 \\
\log \mathrm{t}\end{array}$ & $\begin{array}{c}5 \\
\log \mathrm{t}\end{array}$ & $\begin{array}{c}7,5 \\
\log \mathrm{t}\end{array}$ & $\begin{array}{c}10 \\
\log \mathrm{t}\end{array}$ \\
\hline $\mathbf{8 6}$ & 1,934 & 1,273 & 1,799 & 2,086 & 2,262 & 2,469 & 2,842 \\
57,3 & 1,758 & 0,4248 & 0,977 & 1,345 & 1,596 & 1,921 & 2,080 \\
26,6 & 1,456 & $0,8195-1$ & 0,3729 & 0,729 & 0,989 & 1,352 & 1,604 \\
Wasser & - & $0,3979-1$ & $0,9459-1$ & 0,282 & 0,550 & 0,911 & 1,165
\end{tabular}

Tabelle Ic.

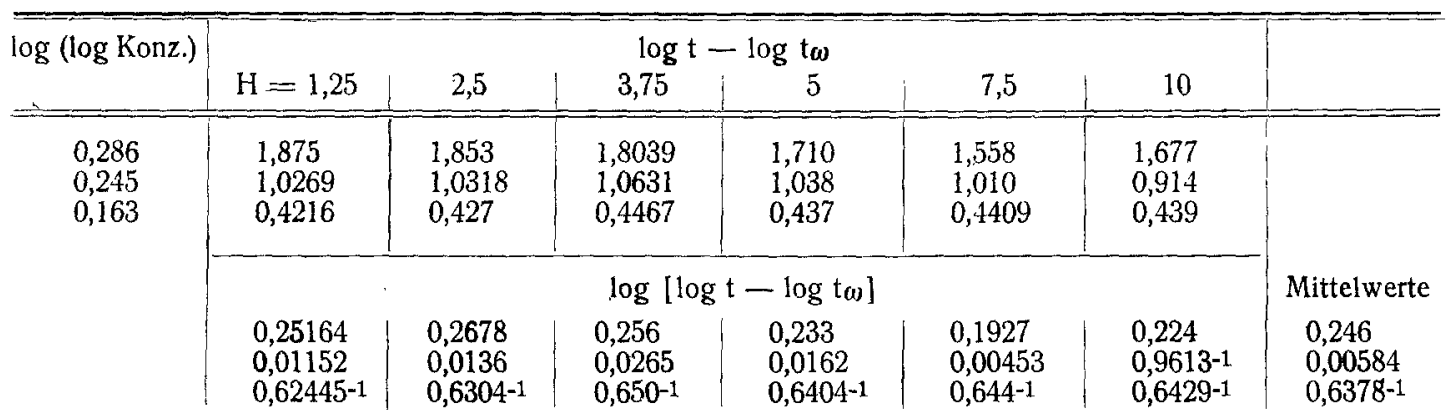

Es ist bemerkenswert, daß die Werte für $\left(\log t-\log t_{\omega}\right)$ bei einer beliebigen Konzentration die gleichen sind für alle Höhenwerte, mithin einen für die betreffende Konzentration charakteristischen Wert liefern [vergl. $\left(\frac{h}{\mathrm{H}-\mathrm{h}}\right)$ bei dem Aufstieg verdünnter Säuren]. Nimmt man die Mittelwerte der $\log \left(\log t-\log t_{\omega}\right)$ und trägt sie auf der Abszisse and die $\log (\log C)$ auf der Ordinatenachse auf, dann liefern die zugeordneten Koordinaten Schnittpunkte, die bemerkenswert genau auf einer geraden Linie liegen, deren Gleichung lautet:

$\log (\log C)=0,244+, 0,2 \log \left(\log t-\log t_{\omega}\right)$ oder $\log C=0,244 .\left(\log t-\log t_{\omega}\right)^{0,2}$

Tabelle Id.

Papier 117.

Konzentration von Glyzerin

\begin{tabular}{l||l|l|l|l|l|l|}
\hline \hline beob- & \multicolumn{5}{|c|}{ berechnet fur $\mathrm{H}=$} \\
achtet & 1,25 & 2,5 & 3,75 & 5 & 7,5 & 10 \\
\hline 86 & 91,2 & 95,5 & 93,3 & 89,1 & 81,2 & 87,1 \\
57,3 & 57,8 & 57,5 & 58,8 & 57,5 & 56,2 & 52,4 \\
28,6 & 29,8 & 29,5 & 30,9 & 30,2 & 30,2 & 30,2
\end{tabular}

Bestimmt man mit Hilfe dieser Gleichung aus der Zeit, welche einerseits Wasser, anderseits die zu untersuchende Lösung brauchen, um eine bestimmte gleiche Höhe zu erreichen, die Konzentration der Lösung, so findet man, wie Tab. Id zeigt, eine in Anbetracht der Fehler gute Uebereinstimmung zwischen Berechnung und Beobachtung.

Die Uebereinstimmung bei Dextrose ist ebenfalls eine relativ gute, wie die analoge Berechnung aus Versuch VIII in folgender Tab. VIIId zeigt.

Tabelle Vllld.

Papier 602 extra hart.

Konzentration für Dextrose in Proz.

\begin{tabular}{|c|c|c|c|c|c|c|}
\hline \multirow{2}{*}{$\begin{array}{l}\text { beob- } \\
\text { achtet }\end{array}$} & \multicolumn{6}{|c|}{ berechnet für $\mathrm{H}=$} \\
\hline & 1,25 & 2,5 & 3,75 & 5 & 7,5 & 10 \\
\hline 100 & 104,2 & 103,3 & 102,1 & 95,9 & 87,3 & $\cdots$ \\
\hline 66,6 & 64,1 & 69,3 & 69,9 & 62,3 & 69,3 & 66 \\
\hline 50 & 40 & 46,8 & 51,2 & 50,8 & 50,8 & 54,7 \\
\hline 33,3 & 35,9 & 36,3 & 36,9 & 36,9 & 37,1 & 38,8 \\
\hline 20 & 19,2 & 23,1 & 24,2 & 24,2 & 24,2 & 27 \\
\hline 12,5 & 10 & 13,6 & 14,5 & 13,8 & 13,1 & 13,4 \\
\hline
\end{tabular}

Die Gleichungen für die Versuche I-VIII, welche in gleicher Weise errechnet die Be- 
ziehung von Konzentration zur Steigzeit wiedergeben, sind in folgender Tab. XIV zusammengestellt.

Tabelle XIV.

$\log$ Konz. $=\boldsymbol{\beta} \cdot\left[\log t-\log t_{(j)}\right]^{\nabla}$

\begin{tabular}{|c|c|c|c|c|}
\hline & & Papier & $\beta$ & $\mathrm{v}$ \\
\hline Glyzerin & $\begin{array}{l}\text { I } \\
\text { III } \\
\text { IV }\end{array}$ & $\begin{array}{l}117 \\
598 \\
602 \text { e.h. } \\
602 \text { h. }\end{array}$ & $\begin{array}{l}1,75 \\
1,75 \\
1,79 \\
1,77 \\
\end{array}$ & $\begin{array}{l}0,2 \\
0,16 \\
0,21 \\
0,16 \\
\end{array}$ \\
\hline Dextrose & $\begin{array}{l}\text { V } \\
\text { VI } \\
\text { VII } \\
\text { VIII }\end{array}$ & $\begin{array}{l}117 \\
602 \mathrm{h.} \\
598 \\
602 \text { e. h. }\end{array}$ & $\begin{array}{l}2,14 \\
2,14 \\
2,14 \\
2,06\end{array}$ & $\begin{array}{l}0,28 \\
0,20 \\
0,32 \\
0,30\end{array}$ \\
\hline
\end{tabular}

Die Gleichungen für Glyzerin sowohl wie für Dextrose weichen untereinander sehr wenig voneinander $a b$, was dafür $z u$ sprechen scheint, daß die Eigenart des Papieres, das zur Messung benutzt wird, nur eine untergeordnete Rolle spielt.

Die Methode ist ein Annäherungsverfahren und theoretisch läbt sich zur Zeit noch nichts über die Natur der Konstanten sagen; es bedarf weiterer exakter Messungen auf diesem Gebiete, bei denen eventuell auch der Einfluß der Temperatur und der Oberflächenspannung, in Rücksicht zu ziehen ist.

\section{$\mathrm{Zus}$ a mmentassung.}

1. Die Steiggeschwindigkeitskonstante $\mathrm{k}$ des kapillaren Aufstiegs ist der Viskosität der Flüssigkeit umgekehrt proportional.

2. Es ist möglich, die Konzentration der Flüssigkeit aus dem Vergleich der Steigzeit derselben mit reinem Wasser angenähert $z u$ berechnen.

\title{
Die „Hysteresis“ wässeriger Aufschwemmungen humoser Böden.
}

\author{
Von Hein rich Puchner (Weihenstephan). (Eingegangen 14. Dezember 1919.)
}

Ueber die "Hysteresis" humoser wässeriger Lösungen ist vom Verfasser ${ }^{1}$ ) bereits früher in dieser Zeitschrift berichtet und dabei betont worden, daß die Flüssigkeit, in der humose Böden aufgeschwemmt sind, durch deren Anwesenheit zur humosen wässerigen Lösung wird. Die Vorgänge, welche sich bei der Hysteresis wässeriger $\mathrm{A}$ u f s ch w e m mungen humoser Böden abwickeln, sind demnach doppelter Art. Einmal unterliegt die humosgewordene Aufschwemmungsflüssigkeit der "Hysteresis" und andererseits sind auch die schwebenden humosen Bodenteilchen daran beteiligt.

Die "Hysteresis" humoser wässeriger Lösungen konnte Verfasser nach den damaligen Untersuchungen in ihrer Wirkung dahin kennzeichnen, daPs sich auf Kosten der gelösten Stoffe unter wahrscheinlicher Mitwirkung niederer Lebewesen eine sich als Niederschlag absetzende Trübung einstellt, welche mineralische und humose Gele sowie kristallin ische Substanz von saurer Beschaffenheit und der Eigenschaft der Flü chtigkeit, der le i chten Löslichkeit in heiBem und schweren Löslichkeit in kaltem Wasser enthält.

Diese Ergebnisse müssen selbstredend mit beriucksichtigt werden, wenn man zur richtigen Beurteilung dessen kommen will, was eintritt, wenn man humose Böden aufschwemmt und die Suspension stellen läßt.

1) H. Puchner, Koll.-Zeitschr. 25, 196 (1919).
Als Versuchsboden wurde wie in den früheren Untersuchungen ein sichtlich dem „Uebergangsmoor" ${ }^{2}$ ) angehörender Torf mit mäBigem, aber stark wechselndem Kalkgehalt benützt. Zur Aufschwemmung kamen je $50 \mathrm{~g}$ des auf einer Knochenmühle zerkleinerten Torfes, welcher zunächst zur allmählichen sicheren Vertreibung der den einzelnen Teilchen anhaftenden Lufthüllen in einer geräumigen Porzellanschale mit Wasser von gewöhnlicher Temperatur in anfangs ganz geringen, nach beginnender Durchfeuchtung immer mehr gesteigerten Mengen geknetet wurde. Darauf folgte zur Trennung der gröberen und feineren Teilchen des Torfbreis voneinander nach weiterer Zufuhr von Wasser ein zweistündiges Kochen, dem sich unter weiterer Verdünnung bis auf eine Gesamtflüssigkeitsmenge von 5 Litérn ein Einfüllen in entsprechend große Glasflaschen anschlob, deren Hals durch einen Gummistopfen verschlossen wurde.

Die unter durchaus gleichartigen Verhältnissen in mehreren Flaschen nebeneinander untergebrachten Aufschlä m $\mathrm{m}$ ungen unterlagen nun in verschiedenen zeitlichen Intervallen der Untersuchung. Beim E in $\mathrm{f} \ddot{\mathrm{l}} \mathrm{ll} \mathrm{e}$ n in die Aufbewahrungsgefäße zeigten die $t r u ̈ b$ e $n$ Flüssigkeiten deutlich saure Reaktion. Aus dem lnhalt der ersten Flasche

2) Unter "Uebergangsmoor" ist hier ein infolge des niederschlagreichen Klimas oberflächlich ausgewaschenes und in hochmoorähnliche Beschaffenheit übergegangenes Niederungsmoor zu verstehen. 